\title{
HDL: o yin-yang da doença cardiovascular
}

\author{
HDL: the yin-yang of cardiovascular disease
}

Camila Canteiro Leança', Marisa Passarelli', Edna R. Nakandakare', Eder C. R. Quintão'

\section{SUMÁRIO}

Estudos epidemiológicos mostram relação inversa entre níveis plasmáticos de HDL-colesterol (HDL-C) e incidência de doença cardiovascular (DCV). O papel antiaterogênico da HDL é atribuído às suas atividades anti-inflamatória, antitrombótica e antioxidante, além de sua participação no transporte reverso de colesterol (TRC), processo pelo qual a HDL remove colesterol dos tecidos periféricos, incluindo macrófagos da íntima arterial, e o transporta para o fígado para ser excretado pela bile. Com base nesses fatos, o HDL-C tornou-se alvo atrativo para a prevenção da DCV. No entanto, o fracasso do torcetrapib, droga que aumenta substancialmente os níveis de HDL-C, em prevenir DCV, além do conhecimento gerado por estudos de modelos animais e doenças monogênicas que afetam a concentração de HDL-C, tem suscitado questionamentos sobre o papel antiaterogênico da HDL. Esta revisão tem como objetivo abordar aspectos atuais do conhecimento da HDL, baseando-se nessas recentes controvérsias. Arq Bras Endocrinol Metab. 2010;54(9):777-84

\section{Descritores}

HDL-colesterol; transporte reverso de colesterol; aterosclerose; proteína de transferência de colesterol éster; torcetrapib; doença de Tangier

\section{SUMMARY}

Epidemiological studies demonstrate an inverse correlation between plasma HDL-cholesterol (HDL-C) concentration and incidence of cardiovascular disease (CVD). The antiatherogenic role of HDL has been attributed to its anti-inflammatory, antithrombotic and antioxidant properties, besides its participation in the reverse cholesterol transport (RCT), whereby cholesterol from peripheral tissues (including macrophages of the arterial intima) is delivered to the liver for excretion in bile. Due to these actions, HDL-C has evolved as an attractive target for prevention of CVD. However, the failure of torcetrapib, drug that substantially increases HDL-C levels, in preventing CVD and data from studies with animal models and with carriers of monogenic disorders affecting HDL-C levels in humans provide conflicting data about HDL being antiatherogenic. This review addresses the current state of knowledge regarding HDL based on these recent controversies. Arq Bras Endocrinol Metab. 2010;54(9):777-84

\section{Keywords}

HDL-cholesterol; reverse cholesterol transport; atherosclerosis; cholesteryl ester transfer protein; torcetrapib; Tangier disease

\author{
Correspondência para: \\ Camila Canteiro Leança \\ Av. Dr. Arnaldo, 455, sala 3305, \\ $3^{\circ}$ andar \\ 01246-900 - São Paulo, SP, Brazil \\ camilacanteiro@gmail.com
}

Recebido em 13/Set/2010 Aceito em 22/Out/2010

\section{INTRODUÇÃO}

$\mathrm{V}$ ários estudos epidemiológicos, entre eles o clássico estudo de Framingham $(1,2)$, revelam que a concentração de HDL-colesterol (HDL-C) é inversamente relacionada à incidência de doença aterosclerótica coronariana. Considerando-se que, atualmente, as doenças cardiovasculares são a principal causa de morte e que estudos clínicos com estatinas revelam risco cardiovascular residual mesmo após correção das concentrações de LDL-colesterol (LDL-C), estratégias farmacológicas para elevar as concentrações de HDL-C tornaram-se alvo de intensas pesquisas. 
O papel antiaterogênico da partícula de HDL tem sido atribuído a uma série de propriedades, entre elas, suas ações anti-inflamatória (3), antitrombótica (4), vasodilatadora e de proteção contra a oxidação das LDLs na parede arterial (5), evento considerado chave na aterogênese. Além disso, um importante mecanismo antiaterogênico da HDL parece relacionado ao transporte reverso de colesterol (TRC). Nesse processo, a HDL remove colesterol dos tecidos periféricos, entre eles os macrófagos da íntima arterial, e o transporta para o fígado, onde ele pode ser excretado na bile e nas fezes (6).

Altas concentrações de HDL-C no plasma têm sido associadas à maior ateroproteção em função de várias ações incluindo o TRC mais eficiente. Entretanto, o estudo de doenças genéticas que afetam as concentrações de HDL-C mostra inconsistências com relação a esse último papel. Além disso, o torcetrapib, inibidor da proteína de transferência de colesterol éster (CETP), capaz de elevar as concentrações de HDL-C em mais de $60 \%$, falhou como terapia redutora de risco cardiovascular (7). Esses fatos têm motivado a busca de um melhor entendimento sobre o metabolismo da HDL.

\section{HDL-C: QUANTIDADE OU QUALIDADE?}

A partir da constatação da relação inversa entre HDL-C e risco de doença coronariana, a concentração de HDL-C passou a ser utilizada em larga escala como um importante indicador laboratorial de risco cardiovascular.

A concentração de HDL-C no plasma é determinada por herança complexa, sendo influenciada por fatores genéticos e ambientais. Sabe-se que peso corporal, tabagismo, exercício físico, consumo de álcool e hábitos alimentares respondem por cerca de $50 \%$ da variação interindividual da concentração de HDL-C na população geral. A hereditariedade contribui para os $50 \%$ restantes. Mutações em genes envolvidos na regulação do metabolismo da HDL, como $A B C A 1$, apoA-I e $L C A T$, estão implicadas em formas raras de hipoalfalipoproteinemia e explicam apenas uma pequena porcentagem desses casos. Por outro lado, polimorfismos genéticos localizados nesses genes são fonte importante de variação no HDL-C (8).

É preciso lembrar que o HDL-C reflete apenas o conteúdo de colesterol presente no total das partículas de HDL e que estas formam um grupo bastante he- terogêneo com relação a tamanho, composição e funcionalidade. A existência de subpopulações distintas de HDL é consistente com o fato de essas partículas exercerem várias funções biológicas (9). O estudo dessas subpopulações tem sido objeto de intensas pesquisas a fim de determinar o papel de cada uma delas no processo antiaterogênico. Com isso, seria possível estabelecer novos biomarcadores de risco cardiovascular mais precisos do que a concentração de HDL-C.

Existem diferentes maneiras de classificar as subpartículas de HDL: por densidade, tamanho, carga e composição (10). O método mais conhecido baseia-se na separação dessas lipoproteínas por densidade, por meio de ultracentrifugação, que permite identificar cinco subfrações de partículas de HDL: HDL-2a, 2b, 3a, $3 \mathrm{~b}$ e $3 \mathrm{c}$. As partículas de HDL-3 são pequenas, densas e pobres em lipídios. Quando comparadas à HDL-2, apresentam maior capacidade de funcionar como aceptoras de colesterol, inibir a expressão de moléculas de adesão nas células endoteliais in vitro e proteger a LDL do estresse oxidativo (5). No entanto, estudos clínicos que investigaram a relação dessas subfrações com risco cardiovascular apresentam resultados divergentes, ora apontando para HDL-2 como preditora de doença cardiovascular $(11,12)$, ora atribuindo esse papel à HDL-3 $(13,14)$. Portanto, até o momento, não há indicação de dosagem das subfrações de HDL para estratificação de risco cardiovascular na prática clínica, uma vez que seu papel não está bem estabelecido (15).

Outro possível marcador de risco cardiovascular é a apoA-I, apolipoproteína mais abundante da HDL, cuja dosagem no plasma, por meio de imunoensaio, é amplamente disponível (16). Estudos apontam para o fato de que a apoA-I tem maior capacidade de predizer doença cardiovascular do que as concentrações de HDL-C (17), mas seu emprego ainda não foi incorporado às principais diretrizes que guiam a prática clínica.

Além da apoA-I, a partícula de HDL carreia uma série de outras apolipoproteínas, entre elas a apoA-II, a segunda mais abundante, apoA-IV, apoC-I, C-II e C-III, apoE e número elevado de proteínas envolvidas no processo de inflamação, imunidade e regulação do sistema do complemento. A importância e a função de cada um desses componentes ainda não estão estabelecidas, mas se sabe que variações na composição proteica das HDLs conferem diferentes funcionalidades a essas partículas (18). Dessa forma, a análise da proteômica dessas lipoproteínas tem se revelado amplo campo de estudo. 


\section{REGULAÇÃO DA CONCENTRAÇÃO INTRACELULAR DE COLESTEROL}

O colesterol é componente de fundamental importância para as células, pois participa de inúmeros processos biológicos, entre eles a formação de membranas, a síntese hormonal e a produção de ácidos biliares necessários à absorção de gordura pelo intestino. As células sintetizam colesterol a partir de acetilCoA e recebem esse esteroide por meio da captação de LDLs e VLDLs pelos receptores de LDL (receptores BE), presentes em domínios específicos da membrana plasmática. Com exceção dos hepatócitos, tecidos esteroidogênicos e intestino, os demais tecidos não são capazes de metabolizar e exportar colesterol em quantidade significante. Por isso, existe um fino mecanismo de regulação da concentração intracelular de colesterol. $\mathrm{O}$ aumento do conteúdo de colesterol na célula leva à diminuição da atividade da enzima 3-hidroxi-3-metil-glutaril-CoA (HMG-CoA) redutase, que tem papel-chave na síntese de colesterol, além da redução da expressão dos receptores de LDL e aumento da atividade da enzima acilcolesterol aciltransferase (ACAT), a fim de estocar colesterol na forma esterificada, uma vez que o excesso de colesterol livre é tóxico para a célula.

O processo de formação da placa aterosclerótica tem início com o acúmulo de colesterol em macrófagos da íntima arterial. Essas células apresentam receptores denominados scavenger que reconhecem partículas de LDL modificadas por glicação, oxidação, carbamilação e associação com imunocomplexos. Quando colesterol entra na célula por meio desses receptores, não há ativação do mecanismo de contrarregulação da entrada de colesterol e ela passa a acumulá-lo, dando origem às chamadas células espumosas $(19,20)$.

Nos macrófagos, o efluxo de colesterol pelas HDLs é crítico para evitar a formação de células espumosas (21). Por essa razão, tem-se buscado estratégias para estimular o transporte reverso de colesterol com a finalidade de reduzir o processo aterogênico (22).

\section{O TRANSPORTE REVERSO DE COLESTEROL}

A formação da partícula de HDL tem início com a secreção de apolipoproteína A-I (apo A-I) pelo fígado, principalmente, e pelo intestino (Figura 1). A apo A-I adquire colesterol (CL) e fosfolípides (FL) via receptores ABCAl (ATP-binding cassette transporter $\mathrm{Al}$ ) no hepatócito, intestino e macrófagos, que exporta lípides através da membrana plasmática. Além disso, a hidrólise de quilomícrons e VLDL pela lipoproteína lipase (LPL) favorece o desprendimento de componentes de superfície dessas lipoproteínas, formando partículas de HDL nascentes ou pré-beta-HDL. Estas ganham colesterol livre e fosfolípides da superfície de células de tecidos extra-hepáticos, como dos macrófagos da parede arterial, também via receptor ABCAl. A enzima lecitina colesterol aciltransferase (LCAT), presente principalmente nas HDL, esterifica o colesterol que migra para o núcleo da partícula, tornando a HDL quase esférica e progressivamente maior (HDL madura). Esse processo de interiorização do colesterol éster na partícula ajuda na manutenção de um gradiente de colesterol livre entre as células e superfície da HDL, que passa a adquirir mais lípides da superfície celular via receptores ABCGI (ATP-binding cassette transporter Gl) e SR-BI (scavenger receptor $\mathrm{BI}$ ).

A CETP medeia troca de triglicérides (TG) de quilomícrons e VLDL por colesterol esterificado (CE) da HDL (Figura 2). A transferência de fosfolípides dessas partículas para HDL ocorre também por ação da proteína de transferência de fosfolípides (PLTP). Como se verá, há muitos trabalhos relacionando aterogênese com a CETP e menos evidência de relação com a PLTP. A partícula de HDL rica em triglicérides é mais sensível à ação da lipase hepática (LH), o que favorece a posterior remoção do colesterol esterificado do interior dessa lipoproteína pelos receptores SR-BI hepáticos. Esta é a chamada via direta do TRC. Neste processo, formam-se remanescentes de HDL, pobres em lípides, que são captados pelo fígado, e apoA-I, que é metabolizada preferencialmente pelos rins via receptores cubilina/megalina.

As partículas de VLDL e LDL, uma vez enriquecidas com colesterol éster proveniente das HDL por ação da CETP, tornam-se mais suscetíveis à ação da lipase hepática e à captação por receptores de LDL presentes na superfície do hepatócito. Essa é a via indireta do TRC, mas que parece representar seu principal componente visto que há estudos mostrando que a maior parte do colesterol que é excretado na bile provém dessa via, apontando para a grande importância da ação da CETP na entrega de colesterol para o fígado (23).

Em resposta ao aumento do conteúdo intracelular de colesterol, o hepatócito reduz sua expressão de receptores de LDL, aumenta a atividade da ACAT, estocando colesterol na forma esterificada, diminui a atividade da enzima HMG-CoA redutase, o que reduz a produção intracelular de colesterol e, simultaneamente, aumenta a síntese de ácidos biliares. 


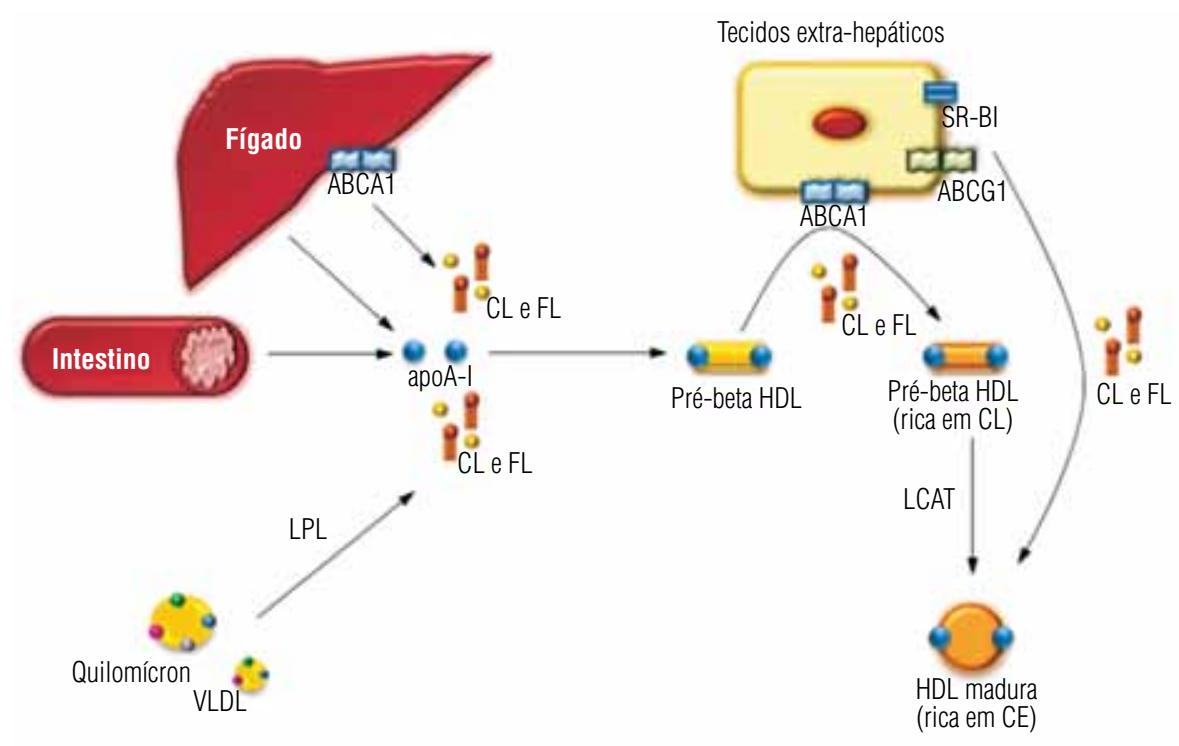

Figura 1. Transporte reverso de colesterol: remoção de colesterol dos tecidos periféricos e formação da HDL madura.

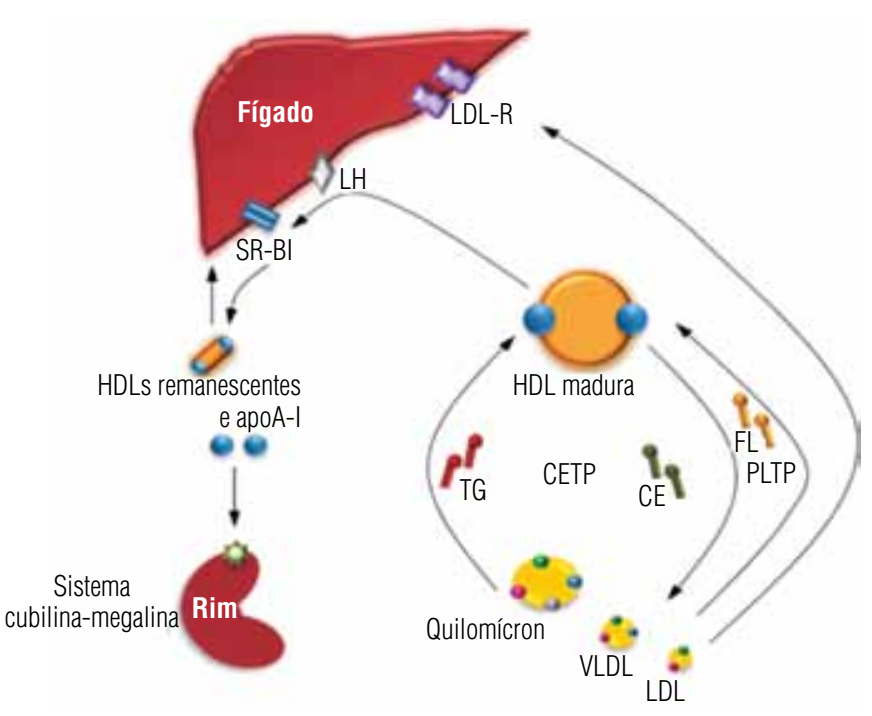

Figura 2. Transporte reverso de colesterol: remoção das partículas de HDL pelo fígado e formação de remanescentes de HDL.

A entrada de colesterol nas células leva à produção de óxidos de colesterol. Estes liberam o complexo LXR/RXR (liver X receptor/retinoid X receptor) de seus repressores, ativando a transcrição dos receptores ABCA-1 e ABCG-1, estimulando, portanto, o efluxo de colesterol. Como já explicado, esse mecanismo é especialmente importante nos macrófagos, incluindo os da parede arterial. Esse fato tem levado à pesquisa de agonistas sintéticos de LXR para prevenção da atero- gênese (24). Os PPARs (peroxisome proliferator-activated receptors) aumentam a expressão de ABCA-1 por induzirem LXR. Ativadores dos PPARs alfa (ex.: fibratos) e gama (ex.: glitazonas) elevam HDL-C por esse mecanismo e têm sido alvo de interesse terapêutico.

Para que o TRC seja eficiente é necessário que todas as etapas do processo estejam íntegras, permitindo o fluxo adequado de colesterol desde sua retirada do macrófago da parede arterial até sua chegada ao fígado para ser excretado na bile.

Não há metodologia estabelecida para medir o TRC em humanos. A maior parte do conhecimento que se tem sobre o assunto vem da observação de doenças monogênicas relacionadas à HDL ou de estudos com modelos animais com ablação gênica ou superexpressão dos receptores envolvidos no TRC (25), ou ainda pela injeção de macrófagos enriquecidos com colesterol radioativo na cavidade peritoneal de animais experimentais, seguindo-se a medida da redistribuição da radioatividade entre plasma, fígado, bile e fezes. Além disso, estudos experimentais e alterações genéticas humanas que determinam reduções severas do HDL-C mostram que a taxa de TRC nem sempre se correlaciona com as concentrações de HDL-C e apoA-I no plasma. Esse fato aponta para a necessidade de desenvolver metodologias que possibilitem a aferição do TRC em humanos, trazendo ganho de informação em relação às medidas isoladas de HDL-C e apoA-I. 


\section{O QUE A GENÉTICA NOS ENSINA SOBRE A HDL}

O estudo de síndromes genéticas que afetam a concentração de HDL-C tem sido importante fonte de conhecimento sobre o metabolismo dessa lipoproteína. A tabela 1 resume as principais causas de hipo e hiperalfalipoproteinemia primárias (familiares).

Tabela 1. Principais causas de hipo e hiperalfalipoproteinemias primárias Hipoalfalipoproteinemias (hipoalfa) primárias

Deficiência de ABCA1 em homozigose (doença de Tangier) ou heterozigose (hipoalfa familiar)

Deficiência de apoA-I

Deficiência de LCAT (Fish-eye disease)

Deficiência de lipoproteína lipase

Hiperalfalipoproteinemias (hiperalfa) primárias

Deficiência de CETP

Deficiência de lipase hepática

Hiperalfa associada à variante apoC-III (Lys58 $\rightarrow$ Glu)

Seria de esperar que doenças relacionadas a valores reduzidos de HDL-C aumentassem o risco de doença cardiovascular prematura, mas isso nem sempre ocorre.

Os primeiros casos da rara doença de Tangier foram descritos no início da década de 1960 por Donald S. Fredrickson (26). Essa doença autossômica recessiva foi o marco inicial dos estudos relacionados à HDL e se caracteriza por concentrações praticamente indetectáveis de HDL-C no plasma, presença de hipertrofia de tonsilas palatinas, hepatoesplenomegalia, hiperplasia dos gânglios linfáticos intestinais e neuropatia. O defeito molecular da doença de Tangier é a mutação em homozigose do receptor ABCA-1, resultando em deficiência completa na expressão dele. Com isso, há prejuízo no efluxo de colesterol celular, levando à deposição de colesterol éster nos tecidos periféricos, e reduzida lipidação das partículas de pré-beta-HDL. As partículas de apoA-I ficam livres na circulação e sofrem rápida catabolização pelos rins (27). Além das baixas concentrações de HDL-C, ocorre trigliceridemia moderadamente elevada, à custa de remanescentes de quilomícrons, de causa desconhecida. Uma possibilidade é que as apolipoproteínas que normalmente são carreadas pela HDL, por estarem livres no plasma, desloquem-se para a superfície dos quilomícrons, dificultando a ação da LPL (28).

É descrito aumento do processo aterosclerótico nos portadores dessa síndrome. No entanto, não se verifica risco cardiovascular tão elevado quanto se esperaria em um quadro de redução tão importante de HDL-C. Há que se levar em consideração que esses indivíduos tam- bém apresentam concentrações diminuídas de LDL-C e isso pode amenizar a aterogênese.

A deficiência de apoA-I, outra causa de hipoalfalipoproteinemia, caracteriza-se por HDL-C baixo e, de maneira geral, correlaciona-se com aumento do risco cardiovascular (29). Existe uma mutação nessa apolipoproteína, denominada apoA-I Milão, em que uma troca de aminoácidos (arginina por cisteína na posição 173) leva à formação de ponte de enxofre entre duas moléculas de apoA-I. Com isso, as partículas de pré-beta-HDL dos portadores tendem a assumir forma mais discoide do que o habitual, possibilitando maior retirada de colesterol da célula. Apesar de os portadores dessa mutação apresentarem HDL-C bastante reduzido (em geral $<20$ $\mathrm{mg} / \mathrm{dL}$ ), não há aumento do risco cardiovascular. Pelo contrário, parece haver proteção devido à maior eficiência do transporte reverso de colesterol nos macrófagos.

O uso da apoA-I recombinante humana para redução da aterosclerose tem sido alvo de intensas pesquisas e seu emprego já se mostrou efetivo em animais (30). Em humanos, a infusão de apoA-I no pós-infarto para redução de placa aterosclerótica mostrou resultados iniciais promissores, mas que precisam ser confirmados em estudo com número maior de pacientes (31).

A deficiência de LCAT é outra situação em que, apesar das concentrações reduzidas de HDL-C (cerca de $20 \mathrm{mg} / \mathrm{dL}$ ), há controvérsias com relação ao risco cardiovascular. A maior parte dos estudos analisou pequeno número de indivíduos e ora aponta para aumento de risco cardiovascular, ora para efeito neutro. Importante ressaltar que os portadores dessa síndrome tendem a apresentar concentrações elevadas de triglicérides, o que pode ser um fator de confusão, uma vez que as lipoproteínas ricas em TG são sabidamente aterogênicas (32). Recentemente, Calabresi e cols. mostraram, em investigação populacional, por meio da medida da espessura íntima-média de carótidas, que indivíduos portadores de deficiência de LCAT não têm aumento de aterosclerose pré-clínica (33).

Modelos animais que superexpressam SR-BI hepático têm redução do HDL-C no plasma e, apesar disso, diminuição na intensidade da aterogênese. Já a ablação gênica desse receptor leva ao aumento da concentração de HDL-C. Paradoxalmente, ocorre intensificação do processo aterosclerótico (34). Até o momento não foi descrita deficiência de SR-BI em humanos e o papel desse receptor na aterogênese permanece incerto.

As síndromes genéticas relacionadas ao metabolismo da HDL são um vasto campo a ser explorado. Boa 
parte dos estudos de que dispomos atualmente analisou número pequeno e/ou heterogêneo de indivíduos, até pela baixa frequência dessas doenças na população. Esse fato pode ser a razão dos resultados muitas vezes conflitantes. Mas não se pode esquecer que essa divergência de resultados também pode ser explicada pela complexidade do metabolismo das lipoproteínas, que é altamente dinâmico e influenciado por inúmeros fatores ambientais e genéticos.

\section{LIÇÕES DO TORCETRAPIB}

O fracasso do primeiro inibidor da CETP testado em humanos, torcetrapib, em reduzir risco cardiovascular mostrou que o metabolismo da HDL é muito mais complexo do que se supunha. O estudo clínico que testou a droga foi precocemente interrompido devido ao aumento da morbidade cardiovascular e mortalidade total (7). Esse resultado pode estar relacionado ao efeito indesejável da droga de aumentar a síntese de aldosterona e induzir hipertensão arterial, mas não se pode descartar que a inibição da CETP tenha efeito deletério sobre o TRC e produza partículas de HDL com menor capacidade funcional (35). De qualquer forma, a partir dessa experiência, a estratégia de elevar as concentrações de HDL-C à custa da inibição da CETP como forma de prevenir doença cardiovascular passou a ser questionada, inclusive pela tendência de aumentar a incidência de câncer e infecção.

O papel da CETP na aterosclerose é ambíguo. Por um lado essa proteína tem papel fundamental na via indireta do TRC, mas o faz à custa do enriquecimento de colesterol em lipoproteínas potencialmente aterogênicas, como as LDL, o que as torna pró-aterogênicas.

A ideia de inibir a CETP como terapia para elevar as concentrações de HDL-C surgiu de estudos com população japonesa portadora de deficiência dessa proteína. Heterozigotos apresentam redução de cerca de $40 \%$ na atividade de CETP e aumento de $30 \%$ no HDL-C. Já os homozigotos têm deficiência completa dessa proteína e aumento de mais de $100 \%$ no HDL-C. As partículas de HDL que predominam nessa condição são as HDL-2. Grandes e ricas em colesterol éster, elas são supostamente menos aptas a funcionar como aceptoras de colesterol e realizar o transporte reverso. As primeiras observações desses genótipos mostravam aumento do risco cardiovascular. De fato, existem relatos de que a inibição completa da CETP pode favorecer a aterogênese na medida em que altera também a composição das lipoproteínas ricas em apoB, tornando-as mais aterogênicas.

No entanto, investigações populacionais mais recentes e de maior peso estatístico apontam para proteção cardiovascular e aumento da longevidade quando a massa e a atividade de CETP estão moderadamente diminuídas (5\%-10\%) (36,37), embora uma metanálise mostre que esse efeito protetor é bastante modesto (38).

Estudos de polimorfismos que elevam a CETP e reduzem as concentrações de HDL-C apontam para aumento do depósito de cálcio nas coronárias, sugerindo aumento do processo aterosclerótico (39).

Investigações em modelos animais também apresentam resultados conflitantes com relação ao papel da CETP na aterogênese. Estudos em coelhos são consistentes em mostrar redução na aterosclerose. No caso de camundongos transgênicos para CETP (estes não expressam naturalmente a proteína), o resultado varia de acordo com o modelo utilizado. Se o clareamento das lipoproteínas ricas em apoB estiver intacto (receptor $\mathrm{LDL}+/+$ ), o processo aterogênico encontra-se diminuído. Caso o clareamento esteja comprometido pela ausência de receptores de LDL, e aumento substancial do LDL-C no plasma, a aterosclerose é aumentada (34).

Em resumo, a CETP e sua relação com a aterogênese tem se mostrado assunto controverso e desafiador, motivando intensas pesquisas nesse campo nos últimos $\operatorname{anos}(40)$.

A CETP não participa apenas da transferência de lipídios. Ela pertence à família das proteínas de ligação a lipopolissacarídeos e de permeabilidade bacteriana e seu papel no sistema imunológico tem sido descrito recentemente.

Camundongos transgênicos para CETP humana submetidos à infusão de lipopolissacarídeo bacteriano produzem menos TNF $\alpha$ e IL-6 em seus macrófagos e apresentam menor mortalidade em comparação com animais selvagens (41). Além disso, esses animais, quando tratados com torcetrapib, desenvolveram mais lesões ateromatosas do tipo inflamatório do que devido ao acúmulo de lipídios na íntima (42).

Por um lado, a CETP parece exercer funções anti-inflamatórias que favorecem o combate contra infecções agudas. No entanto, essa ação pode ser indesejável no ser humano, que, ao atingir uma expectativa de vida muito elevada, torna-se suscetível à aterosclerose pela ação da CETP em elevar as lipoproteínas contendo apoB. 
O fato de a HDL carrear proteínas que têm participação na imunidade e inflamação reforça o conceito de que essa partícula tenha evoluído como parte do sistema imunológico.

\section{VLDL-C E TRIGLICÉRIDES: VILÕES SUBESTIMADOS?}

A grande maioria dos casos de hipoalfalipoproteinemia é devida à combinação com graus diversos de hipertrigliceridemia. Isso decorre do fato de o metabolismo de HDLs e VLDLs estar intimamente relacionado por meio da ação da LPL e CETP, que intermedeiam a troca de componentes entre essas partículas. A associação HDL-C baixo e TG alto no plasma é vista tanto nas causas primárias de hipoalfalipoproteinemia, conforme já citado, quanto nas causas secundárias, cujo maior exemplo é a síndrome metabólica.

Como se sabe, a dislipidemia decorrente do acúmulo de VLDLs e seus remanescentes também é altamente aterogênica (43). Isso torna bastante difícil separar qual o real papel de HDLs e VLDLs no processo aterosclerótico.

$\mathrm{Na}$ tentativa de elucidar esta questão, Frikke-Schmidt e cols. (44) estudaram indivíduos heterozigotos para quatro diferentes mutações inativadoras de $A B C A 1$, com HDL-C variando entre 31 e $50 \mathrm{mg} / \mathrm{dL}$ e concentração de triglicérides normal. Eles não apresentaram risco cardiovascular maior do que controles devidamente pareados com HDL-C normal, variando entre 46 e $73 \mathrm{mg} / \mathrm{dL}$. Esse estudo é um dos pioneiros na tentativa de elucidar o papel do HDL-C isoladamente baixo no risco cardiovascular. Entretanto, há limitações que não permitem extrapolar os resultados para a população geral. Foram estudadas exclusivamente mutações de $A B C A 1$, e esta é apenas uma das causas de hipoalfalipoproteinemia. Além disso, o grupo de indivíduos era formado exclusivamente por caucasianos, o que não permite ampliar a conclusão para outras etnias e, por fim, existe uma série de outros fatores genéticos e ambientais que influenciam o complexo metabolismo lipídico e que precisam ser levados em consideração.

Em resumo, estamos diante apenas da ponta do iceberg. Ainda há muito trabalho pela frente até que se compreenda o real papel da partícula de HDL na aterogênese e o valor da medida isolada do HDL-C como preditor de risco cardiovascular.

Apesar das evidências epidemiológicas e da existência de mecanismos que justificam o papel ateroprotetor da
HDL, estratégias terapêuticas cujo mecanismo de ação baseia-se na elevação da concentração de HDL-C têm se mostrado falhas na redução do risco cardiovascular.

Cada vez mais se valoriza a funcionalidade dessas partículas, e não apenas sua quantidade. Muitas técnicas têm sido desenvolvidas para caracterizar estrutura e função das partículas de HDL e suas subfrações, mas seu uso tem se limitado aos meios acadêmicos, mesmo porque sua relação com desfechos cardiovasculares ainda não está completamente estabelecida.

Finalmente, uma grande questão a ser respondida é se HDL-C e apoA-I são realmente fatores independentes de proteção cardiovascular ou se são apenas marcadores associados a uma complexa condição de alteração do metabolismo lipídico, em que outras lipoproteínas, como as VLDLs, participam de maneira mais importante.

Declaração: os autores declaram não haver conflitos de interesse científico neste estudo.

\section{REFERÊNCIAS}

1. Wilson PW, Abbott RD, Castelli WP. High density lipoprotein cholesterol and mortality. The Framingham Heart Study. Arteriosclerosis. 1988;8(6):737-41.

2. Assmann G, Schulte H, Von Eckardstein A, Huang Y. High-density lipoprotein cholesterol as a predictor of coronary heart disease risk. The PROCAM experience and pathophysiological implications for reverse cholesterol transport. Atherosclerosis. 1996;124:S11-20.

3. Barter PJ, Nicholls S, Rye KA, Anantharamaiah GM, Navab M, Fogelman AM. Antiinflammatory properties of HDL. Circ Res. 2004;95(8):764-72.

4. Mineo C, Deguchi H, Griffin JH, Shaul PW. Endothelial and antithrombotic actions of HDL. Circ Res. 2006;98(11):1352-64.

5. Kontush A, Chantepie S, Chapman MJ. Small, dense HDL particles exert potent protection of atherogenic LDL against oxidative stress. ArteriosclerThromb Vasc Biol. 2003;23(10):1881-8.

6. Lewis GF, Rader DJ. New insights into the regulation of HDL metabolism and reverse cholesterol transport. Circ Res. 2005;96(12):1221-32.

7. Barter PJ, Caulfield M, Eriksson M, Grundy SM, Kastelein JJ, Komajda $\mathrm{M}$, et al. Effects of torcetrapib in patients at high risk for coronary events. N Engl J Med. 2007;357(21):2109-22.

8. LuY, Dollé MET, Imholz S, Van't Slot R, Verschuren WMM, Wijmenga $C$, et al. Multiple genetic variants along candidate pathways influence plasma high-density lipoprotein cholesterol concentrations. J Lipid Res. 2008;49(12):2582-9.

9. Tsompanidi EM, Brinkmeier MS, Fotiadou EH, Giakoumi SM, Kypreos KE. HDL biogenesis and functions: role of HDL quality and quantity in atherosclerosis. Atherosclerosis. 2010;208(1):3-9.

10. Movva R, Rader DJ. Laboratory assessment of HDL heterogeneity and function. Clin Chem. 2008;54(5):788-800.

11. Asztalos BF, Cupples LA, Demissie S, Horvath KV, Cox CE, Batista MC, et al. High-density lipoprotein subpopulation profile and coronary heart disease prevalence in male participants of the Framingham Offspring Study. Arterioscler Thromb Vasc Biol. 2004;24(11):2181-7. 
12. Mueller O, Chang E, Deng D, Franz T, Jing D, Kincaid R, et al. PROCAN study: risk prediction for myocardial infarction using microfluidic high-density lipoprotein (HDL) subfractionation is independent of HDL cholesterol. Clin Chem Lab Med. 2008;46(4):490-8.

13. Asztalos BF, Collins D, Horvath KV, Bloomfield HE, Robins SJ, Schaefer EJ. Relation of Gemfibrozil treatment and high-density lipoprotein subpopulation profile with cardiovascular event in Veterans Affairs High-Density Lipoprotein Intervention Trial. Metabolism. 2008;57(1):77-83.

14. Watanabe $H$, Söderlund S, Soro-Paavonen A, Hiukka A, Leinonen $E$, Alagona C. Decreased high-density lipoprotein (HDL) particle size, prebeta, and large HDL subspecies concentration in Finnish low-HDL families: relationship with intima-media thickness. ArteriosclerThromb Vasc Biol. 2006;26(4):897-902.

15. Krauss RM. Lipoprotein subfractions and cardiovascular disease risk. Curr Opin Lipidol. 2010;21(4):305-11.

16. Forti $\mathrm{N}$, Diament J. Apolipoproteínas B e A-l: fatores de risco cardiovascular? Rev Assoc Med Bras. 2007;53(3):276-82.

17. Van der Steeg WA, Holme I, Boekholdt SM, Larsen ML, Lindahl C, Stroes ES, et al. High-density lipoprotein cholesterol, high-density lipoprotein particle size, and apoliprotein A-I: significance for CV risk: the IDEAL and EPIC-Norfolk studies. J Am Coll Cardiol. 2008;51(6):634-42.

18. Vaisar T, Pennathur S, Green PS, Gharib SA, Hoofnagle AN, Cheung MC, et al. Shotgun proteomics implicates protease inhibition and complement activation in the anti-inflammatory properties of HDL. J Clin Invest. 2007;117(3):746-56.

19. Moore KJ, Freeman MW. Scavenger receptors in atherosclerosis: beyond lipid uptake. Arterioscler Thromb Vasc Biol. 2006;26(8):1702-11.

20. Siqueira AFA, Abdalla DSP, Ferreira SRG. LDL: da síndrome metabólica à instabilização da placa aterosclerótica. Arq Bras Endocrinol Metabol. 2006;50(2):334-43.

21. Tall AR. Cholesterol efflux pathways and other potential mechanisms involved in the athero-protective effect of high density lipoproteins. J Inter Med. 2008;263(3):256-73.

22. Bielicki JK, Zhang H, Cortez Y, Zheng Y, Narayanaswami V, Patel A, et al. A new HDL mimetic peptide that stimulates cellular cholesterol efflux with high efficiency greatly reduces atherosclerosis in mice. J Lipid Res. 2010;51(6):1496-503.

23. Schwartz CC, Van den Broek JM, Cooper PS. Lipoprotein cholesteryl ester production, transfer, and output in vivo in humans. J Lipid Res. 2004;45(9):1594-607.

24. Quinet EM, Basso MD, Halpern AR, Yates DW, Sheffan RJ, Clerin $V$, et al. LXR ligand lowers LDL cholesterol in primates, is lipid neutral in hamster, and reduces atherosclerosis in mouse. J Lipid Res. 2009;50(12):2358-70.

25. Vergeer M, Holleboom AG, Kastelein JJP, Kuivenhoven JA. The HDL hypothesis. Does high-density lipoprotein protect from atherosclerosis? J Lipid Res. 2010;51(8):2058-73.

26. Fredrickson DS, Altrocchi PH, Avioli DS, Goodman DS, Goodman $\mathrm{HC}$. Tangier disease: combined clinical staff conference at the National Institute of Health. Ann Intern Med. 1961;55:1016-31.

27. Nofer JR, Remaley AT. Tangier disease: still more questions than answers. Cell Mol Life Sci. 2005;62(19-20):2150-60.

28. Chung S,Timmins JM, Duong M, Degiloramo C, Rong S, Sawyer $\mathrm{JK}$, et al. Targeted deletion of hepatocyte ABCA1 leads to very low density lipoprotein triglyceride overproduction and low density lipoprotein hypercatabolism. J Biol Chem. 2010;285(16):12197-209.
29. Schaefer EJ, Santos RD, Asztalos BF. Marked HDL deficiency and premature coronary heart disease. Curr Opin Lipidol. 2010;21(4):289-97.

30. Favari E, Gomaraschi M, Zanotti I, Bernini F, Lee-Rueckert M, Kovanen PT, et al. A unique protease-sensitive high density lipoprotein particle containing the apolipoprotein A-I(Milano) dimer effectively promotes ATP-binding Cassette A1-mediated cell cholesterol efflux. J Biol Chem. 2007;282(8):5125-32.

31. Marchesi M, Sirtori CR. Therapeutic use of the high-density lipoprotein protein and peptides. Expert Opin Investig Drugs. 2006;15(3):227-41.

32. Hovingh GK, De Groot E, Van der Steeg W, Boekholdt SM, Hutten BA, Kuivenhoven JA, et al. Inherited disorders of HDL metabolism and atherosclerosis. Curr Opin Lipidol. 2005;16(2):139-45.

33. Calabresi L, Baldassarre D, Castelnuovo S, Conca P, Bocchi L, Candini $\mathrm{C}$, et al. Functional lecithin: cholesterol acyltransferase is not required for efficient atheroprotection in humans. Circulation. 2009;120(7):628-35.

34. Rader DJ, Alexander ET, Weibel GL, Billheimer J, Rothblat GH. The role of reverse cholesterol transport in animals and humans and relationship to atherosclerosis. J Lipid Res. 2009;50:S189-94.

35. Tall AR, Yvan-Charvet $L$, Wang N. The failure of torcetrapib: was it the molecule or the mechanism? Arterioscler Thromb Vasc Biol. 2007;27(2):257-60.

36. Ritsch A, Scharnagl H, Eller P, Tancevski I, Duwensee K, Demetz E, et al. Cholesteryl ester transfer protein and mortality in patients undergoing coronary angiography: the Ludwigshafen Risk and Cardiovascular Health study. Circulation. 2010;121(3):366-74.

37. Schaefer EJ, Asztalos BF. Cholesteryl ester transfer protein inhibition, high-density lipoprotein metabolism and heart disease risk reduction. Curr Opin Lipidol. 2006;17(4):394-8.

38. Thompson A, Di Angelantonio E, Sarwar N, Erqou S, Saleheen D, Dullaart $\mathrm{R}$, et al. Association of cholesteryl ester transfer protein genotypes with CETP mass and activity, lipid levels, and coronary risk. JAMA. 2008;299(23):2777-88.

39. Tsai MY, Johnson C, Kao WH, Sharrett AR, Arends VL, Kronmal $\mathrm{R}$, et al. Cholesteryl ester transfer protein genetic polymorphis$\mathrm{ms}$, HDL cholesterol, and subclinical cardiovascular disease in the Multi-Ethnic Study of Atherosclerosis. Atherosclerosis. 2008;200(2):359-67.

40. Quintão ECR, Cazita PM. Lipid transfer proteins: past, present and perspectives. Atherosclerosis. 2010;209(1):1-9.

41. Cazita PM, Barbeiro DF, Moretti AIS, Quintão ECR, Soriano FG. CETP expression enhances the mouse survival rate in an experimental systemic inflammation model: a novel role for CETP. Shock. 2008;30(5):590-5.

42. De Haan W, De Vries-van der Weij J, Van der Hoorn JW, Gautier T, Van der Hoogt CC, Westerterp M, et al. Torcetrapib does not reduce atherosclerosis beyond atorvastatin and induces more proinflammatory lesions than atorvastatin. Circulation. 2008;117(19):2515-22.

43. Nordestgaard BG, Benn M, Schnohr P. Tybjærg-Hansen A. Nonfasting triglycerides and risk of myocardial infarction, ischemic heart disease, and death in men and women. JAMA. 2007;298(3):299-308.

44. Frikke-Schmidt R, Nordestgaard BG, Stene MCA, Sethi AA, Remaley AT, Schnohr P, et al. Association of loss-of-function mutations in the ABCA1 gene with high-density lipoprotein cholesterol levels and risk of ischemic heart disease. JAMA. 2008;299(21):2524-32. 